Dom. Cien., ISSN: 2477-8818

Vol. 4, núm. 1, enero, 2018, pp. 619-632

\title{
Microempresas en el Ecuador: Caso ciudad de Manta
}

\section{Microenterprises in Ecuador: Case of the city of Manta}

\author{
Miguel Augusto Baque Cantos I \\ miguel.baque@unesum \\ Erick Raúl Baque Sánchez II \\ erick.baque@unesum.edu.ec \\ Gloria Pascuala Chiquito Tigua III \\ gloria.chiquito@unesum.edu.ec \\ Sofía Marianela Baque Parrales IV \\ sophie2918@hotmail.com
}

Recibido: 20 de octubre de 2017 * Corregido: 20 de diciembre de $2017 *$ Aceptado: 01 enero de 2018

I. Ingeniero Comercial, Diplomado en Autoevaluación y Acreditación, Magister en Docencia Universitaria e Investigación Educativa, Docente de la Universidad Estatal del Sur de Manabí, Jipijapa, Ecuador.

II. Ingeniero Comercial, Magister en Contabilidad y Auditoría, Docente de la Universidad Estatal del Sur de Manabí, Jipijapa, Ecuador.

III. Economista, Magister en Contabilidad y Auditoría, Docente de la Universidad Estatal del Sur de Manabí, Jipijapa, Ecuador.

IV. Ingeniera Comercial Mención Comercio Exterior, Universidad Estatal del Sur de Manabí, Jipijapa, Ecuador. 


\section{Resumen}

Las pequeñas y medianas empresas o microempresas nacen con la necesidad de en muchos casos abastecer mercados que no han tenido gran interés por las grandes corporaciones, también nacen con la necesidad de emprendimiento de un grupo de personas que buscan mejorar su calidad de vida generando mayores ingresos que los puedan llevar al desarrollo. Manta es un cantón que pertenece a la provincia de Manabí, es uno de los cantones más importante de esta provincia, posee un puerto por estar ubicado en la costa y una de sus principales actividades económicas es el pesquero a la par de otras actividades como la manufactura, sufrió un duro golpe en su actividad económica y social por el terremoto que los afecto en Abril del año 2016, concentra junto con el cantón de Portoviejo el mayor número de empresas de la provincia con el 48\%. La investigación es de tipo bibliográfica no experimental apoyada por artículos de páginas web y revistas, así como de datos estadísticos aportados por el Instituto Nacional de Estadística y Censos (INEC) sobre información de las microempresas en dicho cantón. Entre las conclusiones más relevantes que se puede llegar después de la investigación, es que es necesario concentrar esfuerzos en divulgar información estadística más precisa y actualizada sobre los comercios y empresas establecidas en el cantón de Manta y su clasificación, apoyadas por las instituciones nacionales y locales.

Palabras claves: Microempresa, Comercio, Actividad, Ingresos, Provincia. 


\begin{abstract}
Small and medium-sized companies or micro-enterprises are born with the need in many cases to supply markets that have not had great interest in large corporations, they are also born with the need for entrepreneurship of a group of people who seek to improve their quality of life by generating higher incomes that can lead them to development. Manta is a canton that belongs to the province of Manabi, is one of the most important cantons of this province, has a port to be located on the coast and one of its main economic activities is fishing along with other activities such as manufacturing, suffered a severe blow to its economic and social activity by the earthquake that affected them in April of 2016, together with the canton of Portoviejo the largest number of companies in the province with $48 \%$. The research is non-experimental bibliographic type supported by articles from web pages and journals, as well as statistical data provided by the National Institute of Statistics and Census (INEC) on information of micro-enterprises in said canton. Among the most relevant conclusions that can be reached after the investigation, is that it is necessary to focus efforts on disseminating more accurate and updated statistical information about the businesses and companies established in the Canton of Manta and its classification, supported by national and local institutions .
\end{abstract}

Keywords: Microenterprise, Commerce, Activity, Income, Province. 


\section{Introducción.}

Se conoce como PYMES al conjunto de pequeñas y medianas empresas que de acuerdo a su volumen de ventas, capital social, cantidad de trabajadores, y su nivel de producción o activos presentan características propias de este tipo de entidades económicas. Por lo general en Ecuador las pequeñas y medianas empresas que se han formado realizan diferentes tipos de actividades económicas entre las que destacamos las siguientes: comercio al por mayor y al por menor, agricultura, silvicultura y pesca, industrias manufactureras, construcción, transporte, almacenamiento, y comunicaciones y otras. (PYMES 2015) (Durán \& Nevares, 2016). Esto nos indica que una microempresa tiene la misma capacidad de realizar cualquier tipo de actividad económica, que una mediana o grande empresa, la diferencia radica en su radio de acción y capacidad instalada, que al final es lo que determina la cantidad de producción que esta puede tener.

Las MYPES se han convertido en uno de los sectores productivos más significativos para las economías de los países emergentes, debido a su contribución en el crecimiento económico y a la generación de riqueza. Las MYPES deben reinventar su modelo de negocio con base en una fuerza productiva que contemple una estructura corporativa y competitiva centrada en la cultura de la planificación, en el talento humano calificado, la flexibilidad y adaptabilidad del negocio, para que puedan sostener sus ventajas empresariales girando en torno a la frontera de la competitividad (Durán \& Nevares, 2016). Esto quiere decir, que por el hecho de que una empresa sea pequeña no se deben olvidar aspectos organizativos que son importantes para su funcionamiento y supervivencia en el mercado, es decir, la microempresas pueden tener la misma estructura que una empresa grande, la única diferencia radica en que es a menor medida pero los preceptos básicos se mantienen. 
El actual contexto económico, social y político de la sociedad ecuatoriana ha generado el surgimiento de nuevas formas productivas. Las contradicciones propias de las sociedades no industrializadas, en las que el crecimiento de la población económicamente activa supera con creces los medios de producción disponibles, ha dejado a un vasto sector de la población al margen del sector moderno o formal de la economía. En el sector de la Microempresa, encontramos un sinnúmero de iniciativas económicas que van desde las menos rentables hasta las más rentables, desde las más transitorias hasta las más estables, desde el comercio, pasando por el servicio y la artesanía, hasta la manufactura; y, lo que es esencial análisis de este trabajo, la incursión en la ejecución de obra pública, a través de las Microempresas que se constituyen y se les otorga personería jurídica justamente para este fin, e intervención en programas de conservación rutinaria de vías (Pazmiño, 2007). Generalmente las microempresas nacen de los sectores informales de la economía, ya que por medio de la transformación de su situación legal tienen la capacidad de poder obtener créditos bancarios y ayuda del Estado para de esta manera ir creciendo poco a poco. En la administración pública es muy común la contratación de microempresas en muchos casos amparados en formas de cooperativa encargadas de ornato público, demarcación de vías, limpieza de calles, entre otras actividades.

Uno de los mayores problemas que tienen las micro, pequeñas y medianas empresas es la dificultad para acceder a los servicios financieros. Las barreras de entrada al sistema financiero formal como: bancos, financieras y mercado de valores, derivan en una serie de problemas que van desde la imposibilidad de impulsar actividades de inversión productiva, de servicios, en capital humano, en tecnología; entre otros; hasta la exclusión y empobrecimiento de grandes segmentos de la población (Alava \& Agustin, 2015). Es normal que esto suceda, los entes financieros deben medir la capacidad de pago que pueden tener las empresas para poder acceder al financiamiento. A su vez 
las condiciones para poder optar a un crédito son burocráticas lo que aleja toda capacidad de estimulación de la microempresa.

Esta situación, limita la posibilidad de incrementar el nivel de ingresos de las familias, y la generación de fuentes de empleo. Por ello la importancia del estudio de las microcréditos en la ciudad de Manta, ya que se ha convertido en una alternativa para el desarrollo económico y por ende social de muchos sectores (Alava \& Agustin, 2015). Si no hay capacidad financiera para arrancar y sostener una microempresa, sucede lo antes mencionado, las pequeñas empresas o las familias que tengan iniciativas de formar una microempresa, primero deben evaluar el mercado donde van a competir, los entes financieros también evalúan los mercados, ya que como se mencionó anteriormente, estas no van a apoyar financieramente una empresa que sus análisis de riesgo les indica que no va a ser rentable, por supuesto esto limita la capacidad de surgimiento y status económico de las familias interesadas en convertirse en emprendedores.

Debido al crecimiento poblacional de Manta, una de las acciones emprendidas por el Gobierno Autónomo Descentralizado, en afán de mejorar la calidad de vida de sus habitantes; ha sido generar un proceso de cambio, concentrándose en acciones para determinar un desarrollo más armónico y planificado del cantón (Alcivar Vélez, 2016). Se comete el error de que a mayor cantidad hay mayor calidad y esto no es cierto, el desarrollo no planificado y controlado termina generando caos, lo que hace generar procesos de estrés en los habitantes y esto desmejora su calidad de vida, es por ellos que los cantones deben generar procesos donde participen los ciudadanos, en donde se escuchen sus planteamientos y se pueda construir un proyecto ciudadano que haga de Manta la ciudad que todos quieren, y aquí es importante el nacimiento de planes de desarrollo 
integrales que logren los objetivos de mejorar calidad de vida, el desarrollo sustentable y el crecimiento sostenido y armónico con la idiosincrasia del Manteño.

\section{Materiales y Métodos}

La investigación está dentro de una metodología de tipo revisión bibliográfica no experimental, en vista que se apoyó en documentos, páginas web y artículos de otros autores, sobre las microempresas ubicadas en la ciudad de Manta, Ecuador. Así como de datos estadísticos aportados por el Instituto Nacional de Estadística y Censo (INEC) de Ecuador de los resultados obtenidos del Censo Nacional Económico realizado en el año 2010.

\section{Resultados}

- Según datos estadísticos presentados en Julio del año 2011 en base a los resultados del Censo Nacional Económico elaborado por el Instituto Nacional de Estadística y Censo (INEC), en la ciudad de Manta existían para la fecha 9.271 comercios, que generaban 1.952 millones de dólares en ingresos por ventas y un total de 39.529 empleados.

- La provincia de Manabí según datos aportados del Directorio de Empresas y Establecimientos del año 2012 del Instituto Nacional de Estadística y Censo (INEC) tenía 62.139 empresas, lo que representa un 9,7\% del total nacional, con un índice de 454 empresas por cada 10.000 habitantes, índice por debajo del nacional que es 470. Si para el censo del año 2011 Manta registraba 9.271 comercios, esto representa un 13,58\% del total de comercios de la provincia de Manabí. Ecuador es un país eminentemente de microempresas, las mismas estadísticas indican que para el año 2012 habían registradas 631.430 microempresas (para el año 2016 la cifra aumenta a 763.636) y esto representa un $89,6 \%$ del total nacional que es de 704.556 empresas. 
Nota: Información actualizada hasta el 2015 indica un total de empresas en Manabí de 67.891 de las cuales el 93,9\% son microempresas. Los cantones de Portoviejo y Manta concentran el mayor número de empresas de la provincia con el $48 \%$, que equivale a 32.653 empresas (INEC). Adicionalmente el $58 \%$ de las grandes empresas se encuentran en esos dos cantones (INEC), esto equivale a 111 empresas grandes de 189 existentes en la provincia (Lopez, 2017).

- Datos aportados por el Informe de Rendición de Cuentas del año 2016, elaborado por el Registro Mercantil de Manta fueron inscritas y certificadas 6.924 nuevas empresas, habiendo una merma en abril del mismo año ocasionado por los terremotos de Manabí y Esmeraldas (Registro Mercantil Manta, 2016).

- Una microempresa es considerada como tal en el Ecuador cuando sus ventas anuales son menores a 100.000\$ y el número de empleados está en el rango de 1 a 9 (Instituto Nacional de Estadistica y Censos, Directorio de Empresas y Establecimientos 2012, 2012). 


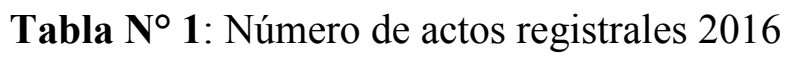

\begin{tabular}{|c|c|c|c|}
\hline Meses & $\begin{array}{l}\text { Numero de } \\
\text { Certificaciones } \\
\text { 2016 }\end{array}$ & $\begin{array}{l}\text { Número } \\
\text { Inscripciones } \\
\mathbf{2 0 1 6}\end{array}$ & $\begin{array}{l}\text { Total Actos } \\
\text { Registrales } \\
\text { 2016 }\end{array}$ \\
\hline Enero & 113 & 610 & $\mathbf{7 2 3}$ \\
\hline Febrero & 180 & 531 & $\mathbf{7 1 1}$ \\
\hline Marzo & 170 & 479 & $\mathbf{6 4 9}$ \\
\hline Abril & 119 & 184 & $\mathbf{3 0 3}$ \\
\hline Mayo & 26 & 142 & $\mathbf{1 6 8}$ \\
\hline Junio & 303 & 541 & $\mathbf{8 4 4}$ \\
\hline Julio & 302 & 399 & $\mathbf{7 0 1}$ \\
\hline Agosto & 256 & 359 & $\mathbf{6 1 5}$ \\
\hline Septiembre & 256 & 411 & $\mathbf{6 6 7}$ \\
\hline Octubre & 169 & $\mathbf{4 . 6 6 2}$ & $\mathbf{5 8 0}$ \\
\hline Noviembre & 195 & 284 & $\mathbf{4 7 9}$ \\
\hline Diciembre & 173 & $\mathbf{6 0 2 4}$ \\
\hline Total & $\mathbf{2 . 2 6 2}$ & & \\
\hline
\end{tabular}

Fuente: (Registro Mercantil Manta, 2016). 
Tabla Nº 2: Clasificación de actividades económicas de la ciudad de Manta.

\begin{tabular}{|l|l|l|}
\hline \multicolumn{1}{|c|}{ Actividades Productivas } & Actividades de Comercio & \multicolumn{1}{|c|}{ Servicios } \\
\hline - Elaboración de & $\begin{array}{l}\text { - Venta al por menor de } \\
\text { alimentos, bebidas y } \\
\text { tabaco }\end{array}$ & $\begin{array}{l}\text { Actividades de restaurantes } \\
\text { servicios móviles de } \\
\text { productos de panadería. }\end{array}$ \\
$\begin{array}{l}\text { - Fabricación de prendas de } \\
\text { vestir }\end{array}$ & $\begin{array}{l}\text { Mantenimiento } \\
\text { reparación de vehículos } \\
\text { automotores. }\end{array}$ & $\begin{array}{l}\text { Actividades de peluquería y } \\
\text { otros tratamientos de belleza. }\end{array}$ \\
\hline
\end{tabular}

Fuente: (Instituto Nacional de Estadistica y Censos, Censo Economico, 2010)

Imagen $\mathbf{N}^{\circ}$ 1: Ingresos generados por sector económico en el cantón de Manta

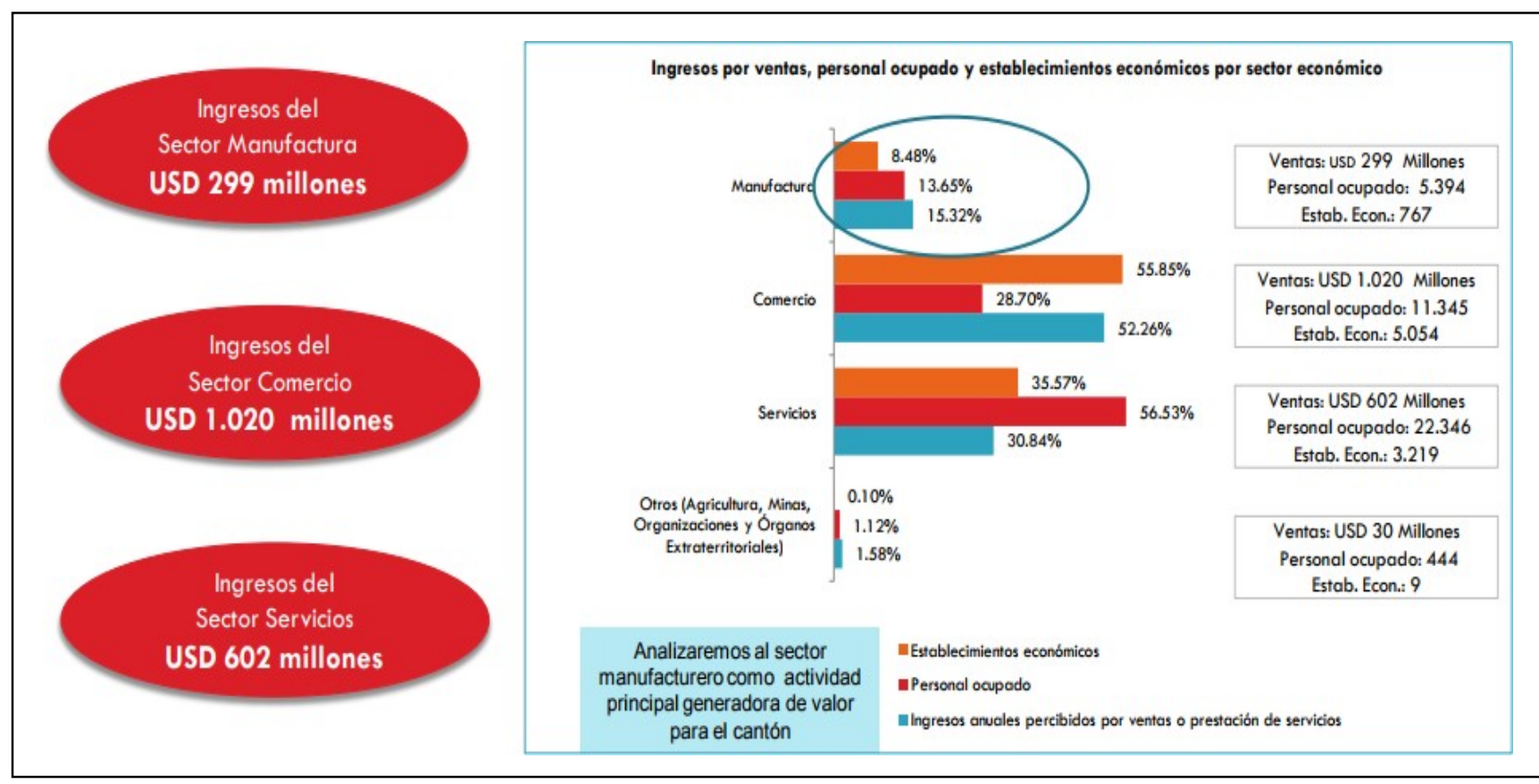

Fuente: (Instituto Nacional de Estadistica y Censos, Censo Economico, 2010) 
Dom. Cien., ISSN: 2477-8818

Vol. 4, núm. 1, Enero, 2018, pp. 619-632

Imagen $\mathbf{N}^{\circ}$ 2: Industria con mayor número de establecimientos en Manta y cuántos ingresos por venta generó
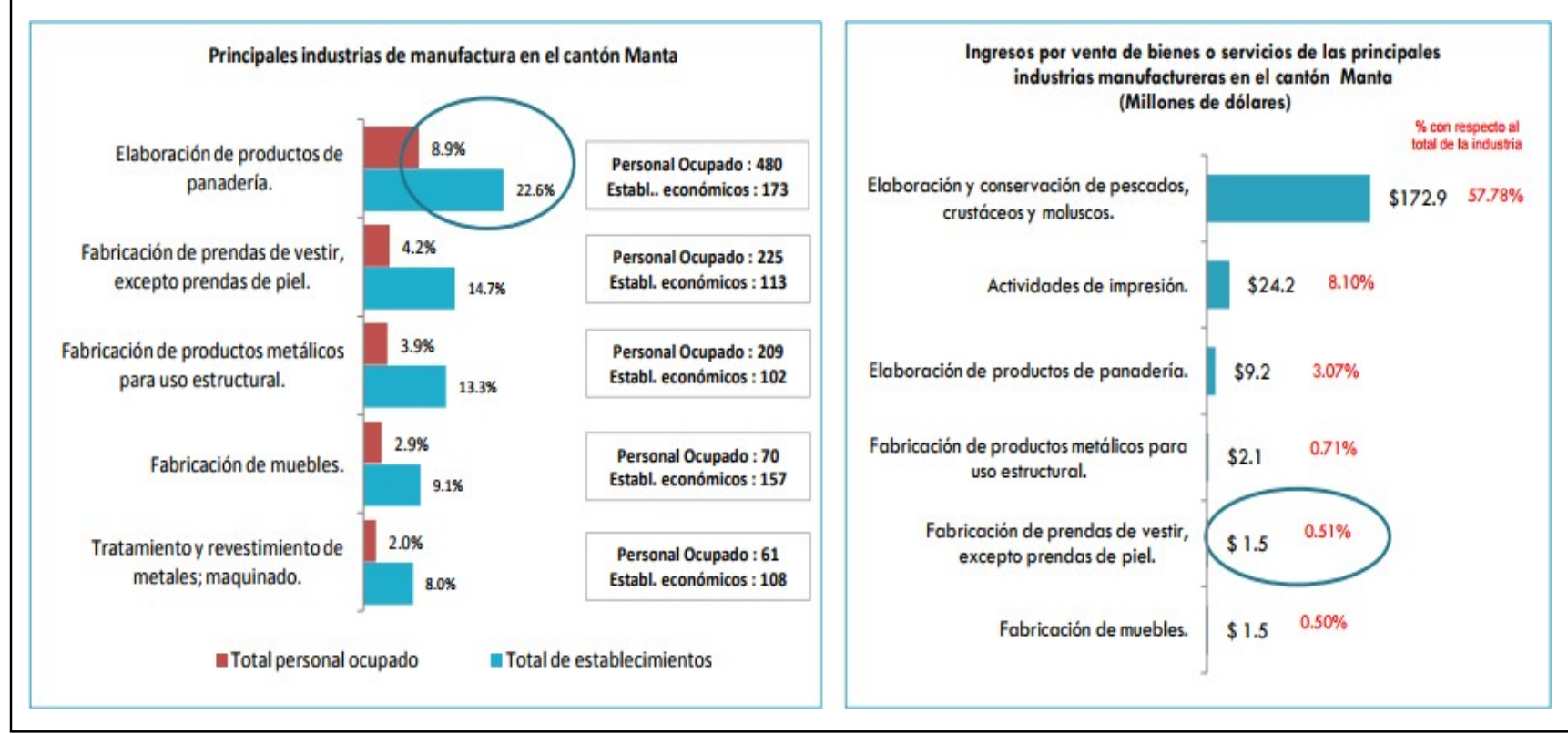

Fuente: (Instituto Nacional de Estadistica y Censos, Censo Economico, 2010).

- Por cantones, Manta reunió el mayor monto de créditos en octubre (41.678.486,27 dólares), el 40 por ciento de la cifra provincial. Le siguió Portoviejo $(\$ 14.697 .103,24)$, cuya cifra equivale al 17 por ciento de Manabí. Los que menos tuvieron son Jama $(\$ 364.104,56)$ y Olmedo $(\$ 151.624,92)$ (Eldiario.ec, 2017). Esto quiere decir que la provincia de Manabí obtuvo un total de \$104.196.215,68 en créditos para las microempresas.

\section{Conclusiones}

- Como conclusión general hay que destacar que a pesar que se consigue información de múltiples variables estadísticas en las páginas oficiales como el Instituto Nacional de Estadística y Censos (INEC) del Ecuador. No hay mucha información actualizada por lo menos hasta el año 2017, se 
consigue información hasta el año 2016 sin embargo se han encontrado algunas discrepancias en cifras que se deben evaluar por los registros oficiales. En casos puntuales es posible conseguir información de algún cantón, de resto la información más precisa se consigue es por provincias, lo que limita la investigación.

- El registro Mercantil de Manta no tiene actualizado su informe de rendición de cuentas hasta la fecha, se consigue información hasta el 2016, sin embargo no se lleva data del tipo de empresa que se registra (microempresa, empresa grande, otros) o por lo menos si la tienen, no la han incorporado.

- La Cámara de Comercio de la Ciudad de Manta no posee registros estadísticos en donde se consiga información actualizada hasta la fecha de la cantidad de empresas que actualmente están funcionando en el cantón y de qué tipo.

- Existe poca información actualizada del apoyo financiero y de créditos para las empresas del cantón de manta, hay información general de la provincia de Manabí en cuanto a créditos otorgados pero las cifras son solo en dólares y no se especifica la cantidad de empresas que obtuvieron los créditos.

- En las páginas oficiales del cantón de manta (Alcaldía de Manta) no hay información estadística sobre la actividad comercial (total de comercios por tipo de actividad y nuevas empresas funcionando). Su informe de rendición de cuentas esta actualizado hasta el año 2016 y no hay información tributaria que pueda dar luces al menos de la cantidad de empresas que pagan impuestos, niveles de morosidad, entre otros datos. 


\section{Recomendaciones}

- Como recomendación general es importante que el gobierno de Manta disponga de una oficina estadística en donde se pueda llevar un registro de datos de todas las actividades económicas y sociales que se generan en el cantón (de tenerla sería importante publicar la data). Muchas veces los institutos nacionales de estadísticas se apoyan en las cifras de los gobierno locales para complementar sus registros, ya que los censos estadísticos necesitan una gran infraestructura y logística para llevarse a cabo y muchas veces no se pueden realizar cada año, es por ello que es necesario la información aportada por aquellas instituciones públicas y privadas (administración tributaria local, cámara de comercio de manta, registro mercantil de manta, otros) que hacen vida en la localidad para tener un verdadero sistema de información estadístico.

\section{Bibliografía.}

Alava, V., \& Agustin, C. (07 de 10 de 2015). Repositorio Universidad de Guayaquil. Obtenido de http://repositorio.ug.edu.ec/bitstream/redug/8658/1/Tesis\%20Ing.\%20Carlos\%20Basurto.pdf

Alcivar Vélez, E. J. (11 de 2016). Repositorio Universidad de Guayaquil. Obtenido de http://repositorio.ug.edu.ec/bitstream/redug/15704/1/Trabajo\%20Titulaci\%C3\%B3n\%20Fina 1.pdf

Durán, M. O., \& Nevares, S. (2016). Talento humano en la microempresa informal. Dominio de las Ciencias, 247-256.

Eldiario.ec. (01 de 12 de 2017). Eldiario.ec. Obtenido de http://www.eldiario.ec/noticias-manabiecuador/456231-manabi-cuarto-en-creditos/

Instituto Nacional de Estadistica y Censos. (2010). Censo Economico.

Instituto Nacional de Estadistica y Censos. (2012). Directorio de Empresas y Establecimientos 2012.

Lopez, H. R. (2017). Estudio del Comportamiento en Compras de Clientes de Manabí en una Empresa de Retail un año después del terremoto. INNOVA Research Journal, 126-134. 
Pazmiño, P. E. (2007). La microempresa en la ejecución de obra pública en el Ecuador . Universidad Andina Simón Bolivar.

Registro Mercantil Manta. (2016). Informe de Rendicion de Cuentas 2016. Registro Mercantil Manta. 